\title{
Basal Cell Adenocarcinoma of the Parotid and Its Simulating Differentials - A Case Report
}

\author{
Kafil Akhtar, Noorin Zaidi, Anjum Ara and Rana K. Sherwani \\ Department of Pathology, J.N. Medical College, Aligarh Muslim University, Aligarh, India \\ Correspondence should be addressed to: Kafil Akhtar; drkafilakhtar@gmail.com \\ Received Date: 23 October 2013; Accepted Date:2March 2014; Published Date: 31 May 2014 \\ Academic Editor: Apostolos A. Epivatianos \\ Copyright (C 2014 Kafil Akhtar, Noorin Zaidi, Anjum Ara and Rana K.Sherwani. Distributed under \\ Creative Commons CC-BY 3.0

\begin{abstract}
Basal Cell Adenocarcinoma (BCAC) is a rare tumour of the salivary glands which has recently been included in the World Health Organization classification. It is thought to be malignant counterpart of basal cell adenoma (BCA) from which it can only be distinguished on the basis of its infiltrative growth pattern and lymphovascular and perineural invasion. Other important tumours like adenoid cystic carcinoma etc also need to be kept in the differential diagnosis. Owing to its rarity, its resemblance to the benign $\mathrm{BCA}$ and the fact that it has to be differentiated from more aggressive tumours, it is important to identify and diagnose BCAC. We thus present a case of BCAC in parotid with a review of literature and a discussion of the important differential diagnoses.
\end{abstract}

Keywords: Parotid gland, Basal Cell Adenocarcinoma.

\section{Introduction}

Basal-cell adenocarcinoma (BCAC) of salivary gland is considered to be the malignant counterpart of basal-cell adenoma (BCA), an established variant of monomorphic adenoma since its segregation from pleomorphic adenoma in 1967.[1,2] Basal cell adenocarcinoma was introduced in the World Health Organization classification of salivary tumors in 1991, and is classified as a lowgrade malignancy in the latest World Health Organization classification (2005). ${ }^{[3}$, ${ }^{4]}$ Although WHO has described BCAC as a tumour with low malignant potential, it has a high recurrence rate. $[2,4,5]$
It is necessary to differentiate basal cell adenocarcinoma from other basaloid cell tumors of the minor salivary glands because of the prognosis and potential differences in treatment, particularly adenoid cystic adenocarcinoma and basaloid squamous carcinoma. ${ }^{[2,5]}$ BCAC resembles $\mathrm{BCA}$ in clinical and histological features, and differentiation is made based on the former's aggressive nature. ${ }^{[6]}$ Very few case reports of basal cell adenocarcinomas have been published in literature. ${ }^{[1,2]}$ Keeping in mind the rarity of $\mathrm{BCAC}$ and the fact that it has to be differentiated from BCA and other basaloidtumours, we present a case of BCAC of parotid gland in a 55 year old female, with a brief review of literature.

Cite this Article as: KafilAkhtar, NoorinZaidi, AnjumAra and Rana K.Sherwani(2014), "Basal Cell Adenocarcinoma of the Parotid and Its Simulating Differentials -A Case Report," JMED Research, Vol. 2014 (2014), Article ID 778687, DOI: 10.5171/2014.778687 


\section{Case Summary}

A 55-year old female presented with a slow growing globular swelling in front of the right ear since 3 years. It was $4 \times 3 \mathrm{~cm}$ in size and firm in consistency, and was partly fixed to underlying structures. Provisional diagnosis of pleomorphic adenoma was made and fine needle aspiration (FNA) was performed, which revealed the presence of cohesive groups of benign looking monomorphic cells along with minimal amount of stromal fragments. The cells were small to round with moderate amount of cytoplasm, and there was no evidence of malignancy like pleomorphism, nuclear overlapping or hyperchromasia.(Figure 1) A tentative diagnosis of monomorphic adenoma was made, and since the patient was elderly and the duration of disease was long, histopathology was advised for final confirmation.

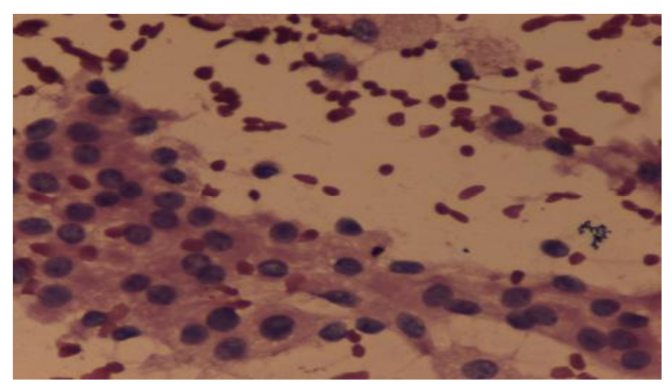

Figure 1: FNAC Smear Showed Cohesive Groups of Benign Looking Monomorphic Cells along with Minimal Amount of Stromal Fragments with Round to Oval Cells with Moderate Amount of Eosinophilic Cytoplasm without Any Evidence of Malignancy Like Pleomorphism, Nuclear Overlapping or Hyperchromasia. H And E X 40

The respected parotid mass, on gross examination was globular and well encapsulated, of size $3 \times 2 \times 1 \mathrm{~cm}$ with solid, homogenous gray cut surface.(Figure 2)
Hematoxylin and Eosin (H\&E) stained sections of the mass showed solid nests of basaloid cells with variable degree of cytological atypia.

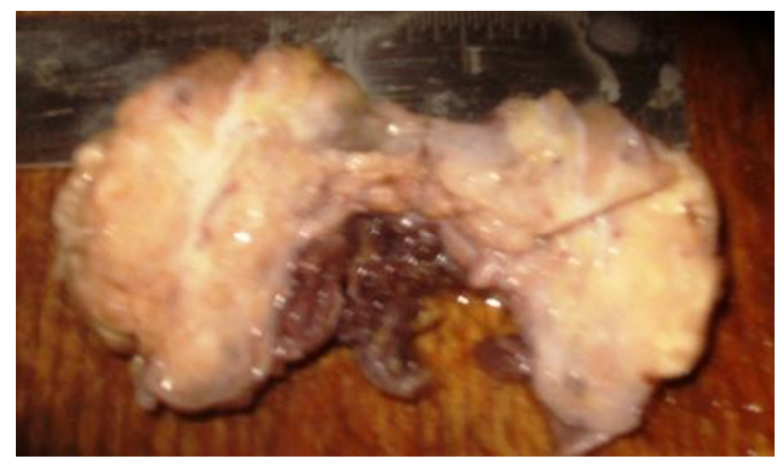

Figure 2: The Resected Parotid Mass, on Gross Examination was Globular and Well Encapsulated, with Solid, Homogenous Gray Cut Surface

Peripheral palisading of dark cells and pale cells with vesicular nuclei and prominent nucleoli were present. Eosinophilic hyaline material distributed as small foci among the tumor cells, or forming a thick band at the periphery of tumor cell nests or cords was also seen. (Figures 3 and 4). 


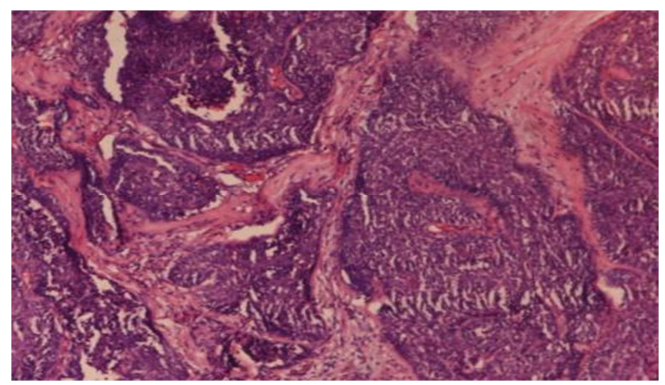

Figure 3: Tissue Section of the Mass Shows Solid Nests of Tumour Cells in Collagenous Stroma.Hematoxylin and Eosin X10

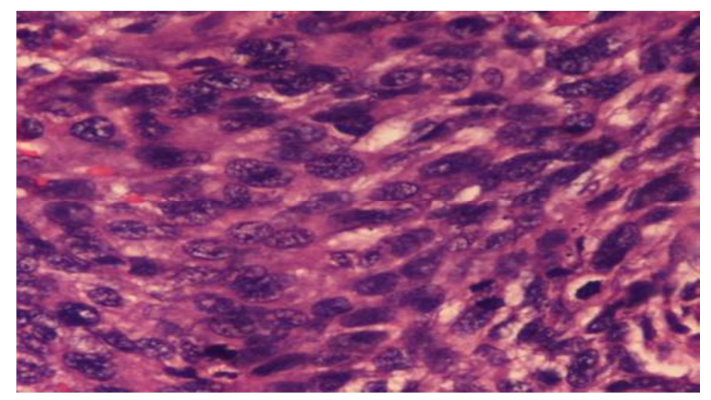

Figure 4: Part of a Solid Nest with Pale and Dark Tumor Cells with Hyperchromatic Nucleus and Prominent Nucleoli with Hyaline Tissue between the Tumor Cells.

Hematoxylin and Eosin X40

Although there was no evidence of vascular or perineural invasion, the tumour showed infiltrative borders with atypical mitosis but without any foci of necrosis. No myoepithelial differentiation was noted in the tumour nests. Immunohistochemical studies showed intense cytoplasmic positivity for vimentin. (Figure 5) Based on these features, a final diagnosis of BCAC, solid variant was made. The patient was administered six cycles of Cisplatin, $50 \mathrm{mg}$ chemotherapy and 50 Gy of Co-60 teletherapy. The patient is in a regular follow-up with no evidence of recurrence, 6 months after resection.

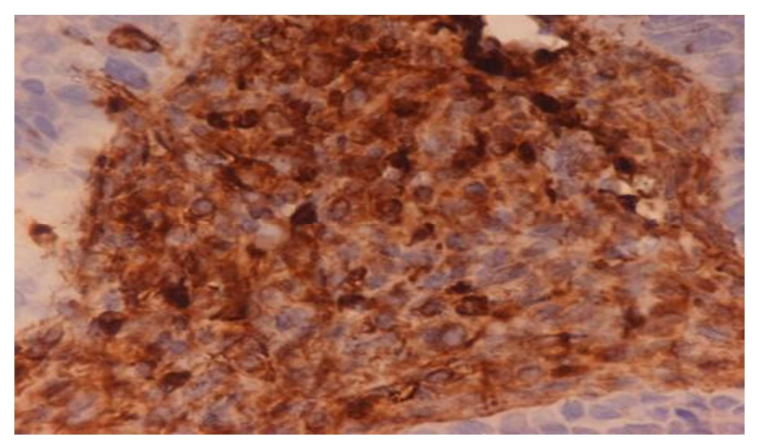

Figure 5: Tumor Nests Showed Intense Cytoplasmic Positivity for Vimentin. Immunostain Vimentin X 40

\section{Discussion}

BCAC of the salivary gland is a rare tumour and recently described entity and comprises $1.6 \%$ of all salivary gland neoplasms, and $2.9 \%$ of all malignant salivary gland neoplasms.[6,7] More than $90 \%$ of cases involve the parotid 
gland.[8]The involvement of the minor salivary glands is very rare, and only few cases have been reported involving the palate, buccal mucosa, labial mucosa, and the submandibular gland. ${ }^{[9]}$ BCACs typically arise in adults above 60 years and have no gender predilection. ${ }^{[5,10]}$ However, according to Nagao et al, there is an age range of 42-81 years (mean 59.2 years).[6] Most tumours are asymptomatic except for swelling, and the duration of tumours before excision ranges from weeks to years. ${ }^{[4]}$

The histogenesis is still unknown and although some authors propose it may develop from a preexisting basal cell adenoma; most, however, believe it is a de novo lesion. ${ }^{[4,5]}$ According to some authors it appears to arise from pluripotent ductal reserve cells. ${ }^{[2,11]}$ The cut surface varies from grey, tan-white to brown in colour with a homogeneous texture. ${ }^{[4]}$ According to the latest WHO classification, four histomorphological patterns are foundsolid, membranous, tubular and trabecular, out of which solid pattern is the most frequently encountered.[1,4,5,6,11] This pattern consists of dark and pale basaloid cells forming variable sized and shaped nests, separated by thin septa or thick bands of collagenous stroma. ${ }^{[4]}$ In our case the gross appearance was homogenous gray with solid histological pattern.

Some authors have tried to differentiate BCAC from BCA on cytological examination; but others believe that they have similar architectural and cytologic features.[2,5] Therefore, the diagnosis of these tumours must be made histologically.[11] According to a study, neither ultrastructural characteristics nor immunohistochemistry findings appear to distinguish basal cell adenocarcinoma from basal cell adenoma.[12] The main features favoring BCAC over BCA are infiltrative growth pattern, vascular and perineural invasion, cellular atypia, necrosis and mitosis. $[1,2,5,6]$ True invasion must be distinguished from both multinodularity with a pushing type of growth pattern and a multifocal origin in adjacent salivary lobules, both of which are features of basal cell adenoma. [9] Histological picture of our case showed a presence of a cytological atypia and infiltrative growth pattern although there was no evident vascular or perineural invasion.

Apart from BCA, BCAC needs to be differentiated from the solid variant of adenoid cystic carcinoma (ACC). An important point to be born in mind is that, with BCAC only wide surgical excision is required, unlike adenoid cystic carcinoma which requires an extensive and aggressive surgical approach.[11] Histologically, the presence of dark hyperchromatic angulated nuclei in ACC are in contrast with the vesicular nuclei of BCAC; prominent peripheral palisading of the outer layer in BCAC is also an important differentiating feature.[11] BCAC also lacks the cribriform pattern, pseudo-cysts of amorphous and, basophilic material characteristic of ACC. [5]

Another important differential diagnosis is basaloid squamous cell carcinoma (SCC). Basaloid SCC is usually located in the hypopharynx, base of tongue, and supraglottic larynx, regions in which basal cell adenocarcinoma is rarely found. ${ }^{[8]}$ Unlike BCAC, basaloid SCC shows squamous differentiation that involves the mucosal epithelium, which may demonstrate dysplasia, carcinoma in situ, or invasive squamous cell carcinoma. ${ }^{[5]} \mathrm{Also}$ the specific immunohistochemical markers of keratin 7, vimentin and S-100 protein are positive in BCAC but not in BSCC, which are helpful in the differential diagnosis of these tumors.[12]

While they are locally destructive and often recur, BCACs only occasionally metastasize, and death of patients is rare. ${ }^{[4]}$ Therefore most authors advocate surgical excision with wide resection margins and close follow-up.[2, 5, 8,11] Radiotherapy has been proposed for lesions in the minor salivary glands, and for tumors with a diffuse infiltrating pattern to adjacent tissue. $[5,8,9,11]$

\section{Conclusion}

BCAC is a newly identified and rare tumour of salivary gland which has to be differentiated from its benign counterpart 
and from more aggressive similar appearing malignant tumours. Although it is a slow growing tumour with low malignant potential, it has a high rate of recurrence. Therefore, a possibility of BCAC should always be kept in mind while dealing with cases of salivary gland neoplasms, for proper management of the patient.

\section{References}

1. Ellis, G. L. \& Wiscovitch, J. G. (1990). "Basal Cell Adenocarcinomas of the Major Salivary Glands," Oral Surgery, Oral Medicine, Oral Pathology, 69:461469.

2. Mardi, K., Kaushal, V. \&Asotra, S. (2011). "Basal Cell Adenocarcinoma of Submandibular Salivary GlandProblems in Cytologic Diagnosis," Journal of Cytology, 28:203-206.

3. Seifert, G., Sobin, L. H. \&Thackray, A. C. (1991). Histological Typing of Salivary Gland Tumours, 2nd Edn, Berlin, Germany: Springer-Verlag; World Health Organization International Histological Classification of Tumours,Pp 9-10.

4. Barnes, L., Eveson, J. W., Reichart, P. \&Sidransky, D. (2005). "Pathology and Genetics of Tumours of the Head and Neck," Lyon, France: IARC Press;Vol 9, World Health Organization Classification of Tumours, Pp 229-230.

5. Farrell, T. \& Chang, Y. L. (2007). "Basal Cell Adenocarcinoma of Minor Salivary Glands," Archives of Pathology \& Laboratory Medicine, 131:1602-1604.

6. Nagao, T., Sugano, I., Ishida, Y., Hasegawa, M., Matsuzaki, O. \& Konno, A. (1998). "Basal Cell Adenocarcinoma of the Salivary Glands: Comparison with Basal Cell Adenoma through Assessment of Cell Proliferation, Apoptosis, and Expression of P53 and Bcl-2," Cancer, 82: 439-447.
7. Sharma, R., Saxena, S. \& Bansal, R. (2007). "Basal Cell Adenocarcinoma: Report of a Case Affecting the Submandibular Gland," Journal of Oral \& Maxillofacial Pathology, 11: 56-59.

8. Parashar, P., Baron, E., Papadimitriou, J., Ord, R. \&Nikitakis, N. (2007). "Basal Cell Adenocarcinoma of the Oral Minor Salivary Glands: Review of the Literature and Presentation of Two Cases," Oral Surgery, Oral Medicine, Oral Pathology, Oral Radiology and Endodontology, 103:77-84.

9. Jayakrishnan, A., Elmalah, I., Hussain, K. \& Odell, E. W. (2003). "Basal Cell Adenocarcinoma in Minor Salivary Glands," Histopathology, 42: 610-614.

10. Muller, S. \& Barnes, L. (1996). "Basal Cell Adenocarcinoma of the Salivary Glands: Report of Seven Cases and Review of the Literature," Cancer, 78: 2471-2477.

11. Ward, B. K., Seethala, R. R., Barnes, E. L. \& Lai, S. Y. (2009). "Basal Cell Adenocarcinoma of a Hard Palate Minor Salivary Gland: Case Report and Review of the Literature," Head \& Neck Oncology, 1:41-44.

12. Andreadis, D., Nomikos, A., Epivatianos, A., Poulopoulos, A. \&Barbatis, C. (2005). "Basaloid Squamous Cell Carcinoma versus Basal Cell Adenocarcinoma of the Oral Cavity," Pathology; 37(6):560-563. 